\title{
WHAT IS THE DIFFERENCE? BLAZHKO AND NON-BLAZHKO RRab STARS AND THE SPECIAL CASE OF V123 IN M3
}

\author{
J. Jurcsik ${ }^{1}$, P. Smitola ${ }^{1}$, G. Hajdu ${ }^{2,3}$, C. Pilachowski ${ }^{4,11}$, K. Kolenberg ${ }^{5,6}$, Á. Sódor ${ }^{1,7}$, G. Fû́RÉsz ${ }^{6}$,

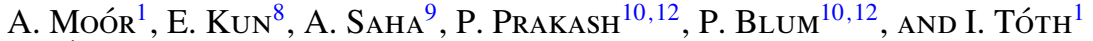 \\ ${ }^{1}$ Konkoly Observatory, H-1525 Budapest, P.O. Box 67, Hungary; jurcsik@konkoly.hu \\ ${ }^{2}$ Instituto de Astrofísica, Pontificia Universidad Católica de Chile, Av. Vicuña Mackenna 4860, 782-0436 Macul, Santiago, Chile \\ ${ }^{3}$ The Milky Way Millennium Nucleus, Av. Vicuña Mackenna 4860, 782-0436 Macul, Santiago, Chile \\ ${ }^{4}$ Department of Astronomy, Indiana University Bloomington, Swain West 319, 727 E. 3rd Street, Bloomington, IN 47405, USA \\ ${ }^{5}$ Harvard-Smithsonian Center for Astrophysics. Astronomy, 60 Garden Street MS-42, Cambridge, MA 02138, USA \\ ${ }^{6}$ Instituut voor Sterrenkunde, K.U. Leuven, Celestijnenlaan 200D, B-3001 Heverlee, Belgium \\ ${ }^{7}$ Royal Observatory of Belgium, Ringlaan 3, B-1180 Brussel, Belgium \\ ${ }^{8}$ Department of Experimental Physics and Astronomical Observatory, University of Szeged, 6720 Szeged, Dóm tér 9, Hungary \\ ${ }^{9}$ National Optical Astronomy Observatories, Tucson, AZ 85726-6732, USA \\ ${ }^{10}$ The Smithsonian Astrophysical Observatory, Cambridge, MA 02138, USA \\ Received 2013 September 5; accepted 2013 September 20; published 2013 November 12
}

\begin{abstract}
In an extended photometric campaign of RR Lyrae variables of the globular cluster M3, an aberrant-light-curve, non-Blazhko RRab star, V123, was detected. Based on its brightness, colors and radial-velocity curve, V123 is a bona fide member of M3. The light curve of V123 exhibits neither a bump preceding the light minimum, nor a hump on the rising branch, and has a longer than normal rise time, with a convex shape. A similar shape characterizes the mean light curves of some large-modulation-amplitude Blazhko stars, but none of the regular RRab variables with similar pulsation periods. This peculiar object thus mimics Blazhko variables without showing any evidence of periodic amplitude and/or phase modulation. We cannot find any fully convincing answer to the peculiar behavior of V123, however, the phenomenon raises again the possibility that rotation and aspect angle might play a role in the explanation of the Blazhko phenomenon, and that some source of inhomogeneity (magnetic field, chemical inhomogeneity) deforms the radial pulsation of Blazhko stars during the modulation.
\end{abstract}

Key words: globular clusters: individual (M3) - stars: individual (M3 V123) - stars: oscillations (including pulsations) - stars: variables: RR Lyrae - techniques: photometric - techniques: radial velocities

Online-only material: color figures

\section{INTRODUCTION}

RR Lyrae variables are thought to be well-known objects, both from evolutionary and pulsation points of view. They are horizontal-branch (HB) stars pulsating in the radial fundamental or in the first overtone mode, and some of them (the double-mode RR Lyrae stars) in both. However, the light variation of about $50 \%$ of the RRab stars is not stable; they exhibit periodic or complex phase and amplitude modulations (the Blazhko effect, Blazhko 1907).

The light curves of the non-modulated, single-mode RR Lyrae stars are quite regular as the interrelations among their Fourier parameters (Jurcsik \& Kovács 1996) indicate. The applicability of the relations between the physical parameters and lightcurve parameters (Jurcsik 1998; Kovács \& Walker 2001, and references therein) verify that the pulsation light curves of the variables reflect their physical properties. Consequently, light curves of RR Lyrae variables with similar physical parameters are similar, and vice versa. Hydrodynamical modeling of the pulsation light curves of RR Lyrae stars has also been successful (Marconi \& Degl'Innocenti 2007).

Some RR Lyrae stars show, however, somewhat anomalous light variations. In some cases, a discrepant evolutionary state is behind the anomalous behavior of these stars. The triplemode variable AC Andromedae proved to be a large-mass object

\footnotetext{
${ }^{11}$ The WIYN Observatory is a joint facility of the University of Wisconsin-Madison, Indiana University, Yale University, and the National Optical Astronomy Observatory.

12 Summer interns.
}

evolving off the main sequence (Fitch \& Szeidl 1976; Kovács \& Buchler 1994). Quite recently, Pietrzynski et al. (2012) found a very low, $0.26 M_{\odot}$, "RR Lyr"-like pulsator in a binary system, where mass transfer influenced the stellar evolution of the components. However, it is not at all obvious how to detect the anomalous behavior of the light curve of mono-periodic RR Lyrae stars. In a heterogeneous sample of different age, mass, and chemical composition, all of these parameters influence the shape of the light variation. Therefore, homogeneous groups of objects are ideal targets for finding anomalous variables, such as variables in, e.g., globular clusters.

In the present Letter, a mono-periodic RR Lyrae star with an anomalous light-curve shape in M3 (V123) is displayed and its possible connection with the Blazhko effect is discussed.

\section{OBSERVATIONS}

One of the most RR Lyrae rich globular clusters, M3, was extensively observed with the $90 / 60$ Schmidt telescope of the Konkoly Observatory in 2012. The data set covers a 200 day long period, and it contains about 1000 measurements in each of the Johnson-Cousins $B, V, I_{\mathrm{C}}$ photometric bands. Light curves of variables excluding the most crowded regions were obtained from both aperture photometry and image subtraction method techniques.

Spectroscopic observations were obtained using the Hydra fiber spectrograph on the $3.5 \mathrm{~m}$ WIYN telescope at Kitt Peak with a resolving power of $R=3600$ in 2000 (Sandstrom et al. 2001). A $1000 \AA$ region, centered on the Mg I triplet 

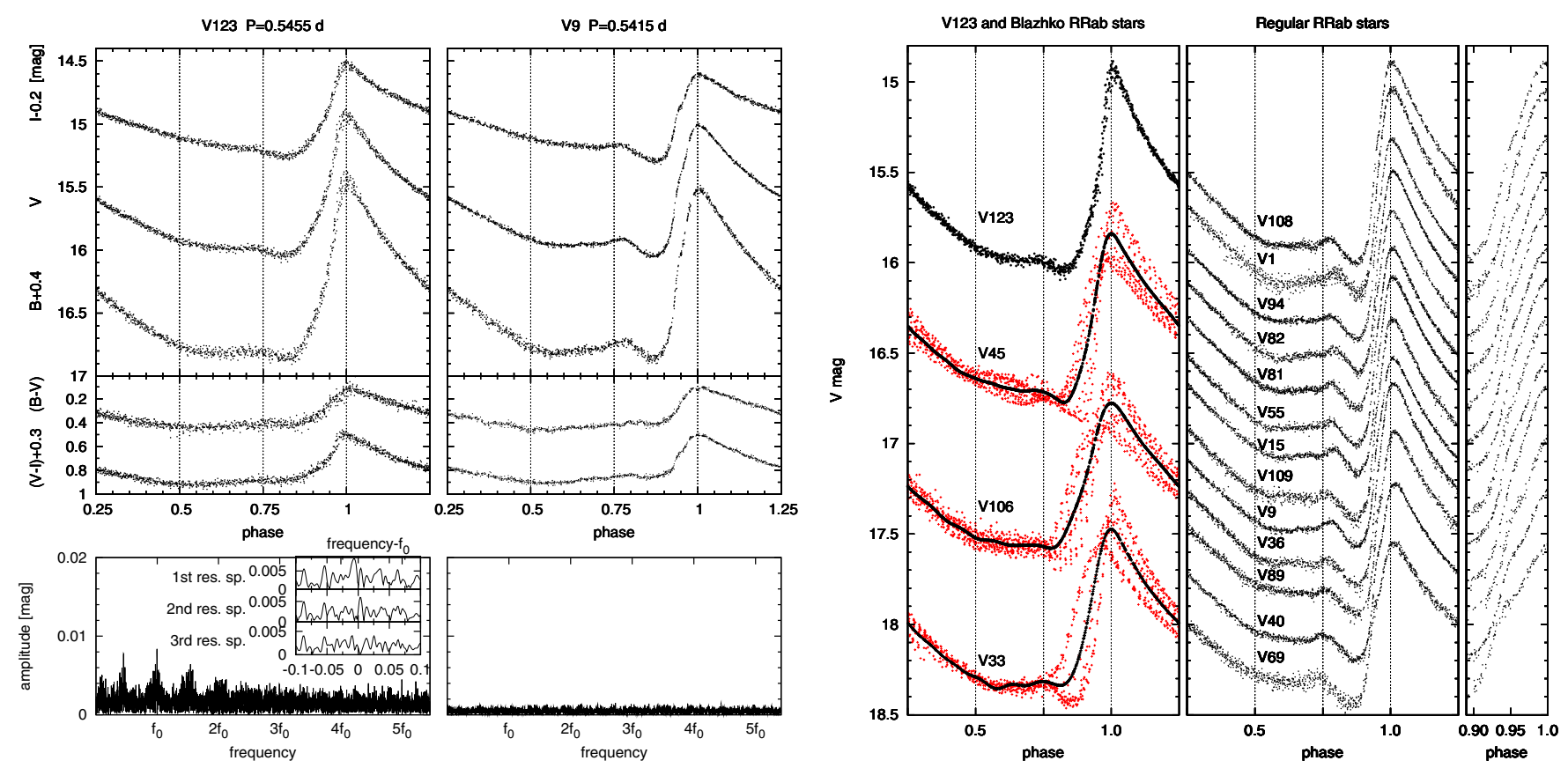

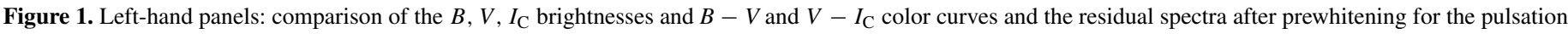

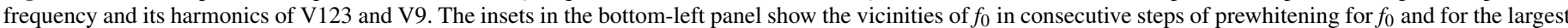

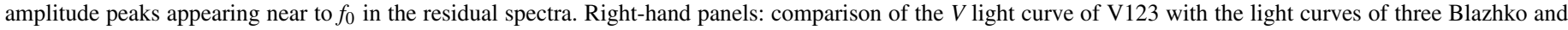

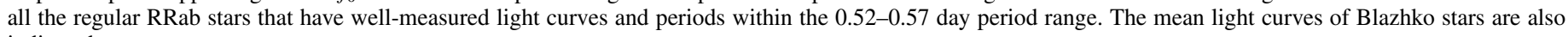
indicated.

(A color version of this figure is available in the online journal.)

lines (5180 ̊) was selected for observation both because of the suitability of the triplet lines for radial-velocity determinations, and because of the relative brightness of the RR Lyrae stars at this wavelength.

Radial velocities for the variables and red giants were measured using the IRAF ${ }^{13}$ task fxcor. Velocities were determined by cross-correlation relative to the twilight sky spectrum observed with the same instrument. The spectral region for crosscorrelation was restricted to $\lambda \lambda 5000-5400$ to avoid $\mathrm{H} \beta$.

The typical uncertainties of the radial-velocity values of variables and giants are $12 \mathrm{~km} \mathrm{~s}^{-1}$ and $1 \mathrm{~km} \mathrm{~s}^{-1}$, respectively, while the uncertainty of the radial-velocity zero point is estimated to be less than $1 \mathrm{~km} \mathrm{~s}^{-1}$.

The photometric and radial-velocity data and full details of the reduction and calibration processes will be given in J. Jurcsik et al. (in preparation).

\section{V123 IN M3}

In the course of analyzing the data, we noticed that the light curve of V123 $\left(V=15.70, \alpha_{2000}=13^{\mathrm{h}} 41^{\mathrm{m}} 52^{\mathrm{s}} .30\right.$, $\delta_{2000}=+28^{\circ} 06^{\prime} 04^{\prime \prime} .33, P=0.5455$ days), a non-Blazhko star, is atypical compared to the light curves of similar-period RRab stars.

The light and color curves of V123 are compared to the light variation of V9 ( $P=0.5414$ days), a typical-light-curve-shape RRab with similar period, in the left panels of Figure 1. The differences are evident: in V123, the bump on the lower part of the descending branch is marginal, and there is no hump on

\footnotetext{
13 IRAF is distributed by the National Optical Astronomy Observatories, which are operated by the Association of Universities for Research in Astronomy, Inc., under cooperative agreement with the National Science Foundation.
}

the rising branch, while a pronounced bump and a minor hump characterize the light curve of V9. The rising branch of V123 has an anomalous convex shape, it is less steep and the length of the rise time from minimum to maximum is longer than in other RRab stars.

Although both V123 and V9 are well-measured, separated stars, the scatter of the light curves of the two stars is obviously different. The scatter of the light curves of V123 arises from minor night to night variations, which can be clearly detected in the variation of the heights of the maximum brightnesses. Nevertheless, the residual spectrum of V123 shown in the bottom-left panels of Figure 1 does not show the typical features of a Blazhko star: similar equidistant multiplet-frequency structures separated by a well-defined modulation frequency at the pulsation frequency, and its harmonics. Instead, although the most prominent residual frequencies appear at $f_{0}$, their separations are not equidistant, consequently they do not reflect a regular modulation of the light curve. No signal with an amplitude larger than $2 \mathrm{mmag}$ at any half-integer frequency or at the possible positions of the first and second overtone modes is detected.

The $V$ light curves of V123 and three strongly modulated Blazhko stars with similar pulsation periods (V33, $P=0.5252$ days; V45, $P=0.5369$ days; and V106, $P=0.5469$ days), and all the regular RRab stars in our sample with periods between 0.52 and 0.57 days are shown in the right-hand panels of Figure 1. The mean light curves of the Blazhko stars, defined by the pulsation components of the Fourier solution including both pulsation and modulation components, are set out. The figure clearly shows that: (1) all the RRab stars plotted in the right panel exhibit very similar light-curve shapes; and (2) the light curve of V123 resembles the mean light curves of Blazhko stars much more than the light curves of normal RRab stars. We 

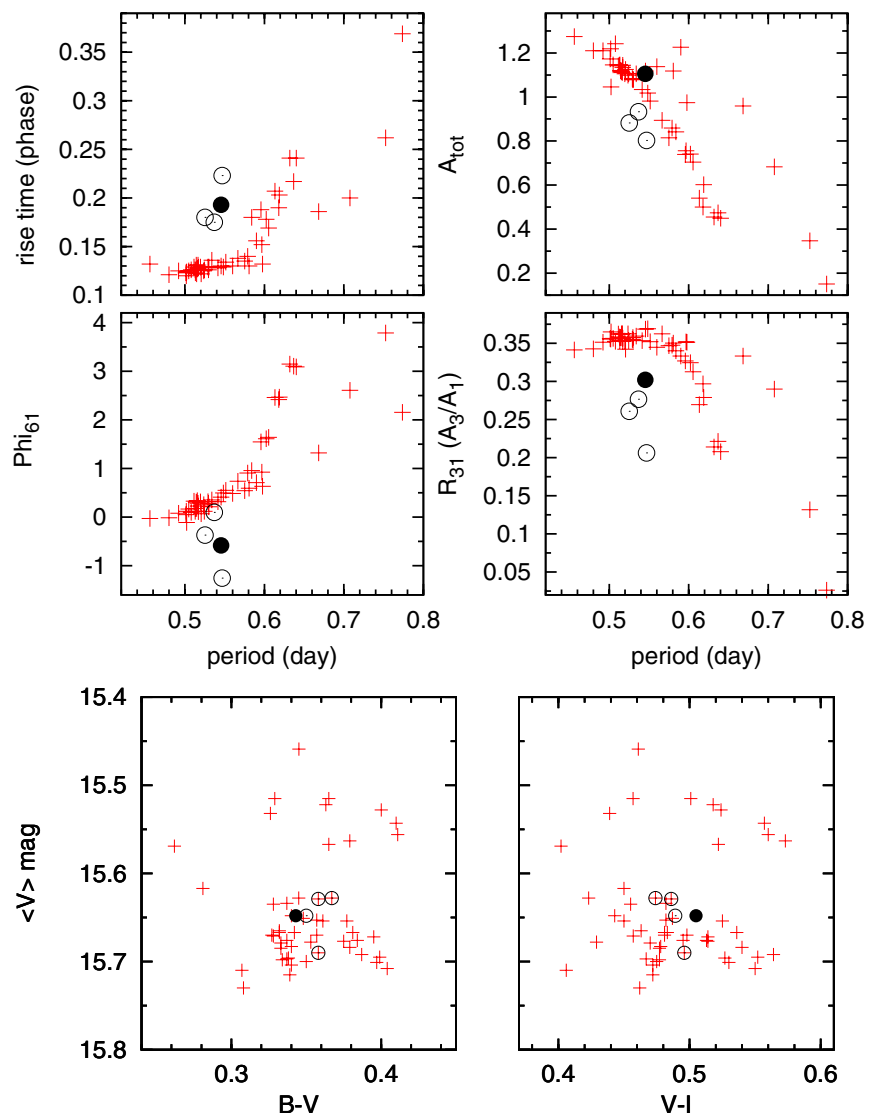

Figure 2. Rise time, $A_{\text {tot }}, R_{31}$, and $\Phi_{61}$ Fourier parameters of the $V$ light curves of M3 RRab stars are shown in the top and middle panels. Regular variables, V123, and the parameters of the mean light curves of three Blazhko stars shown in Figure 1 are denoted by the "+" symbol, filled, and open circles, respectively. Intensity-averaged $V$ magnitudes of regular RRab stars in M3 vs. magnitudeaveraged $B-V$ and $V-I$ colors are plotted in the bottom panels. The positions of V123 and four variables with similar pulsation periods (V9, V36, V4, and V89) are shown by filled and open circles, respectively.

(A color version of this figure is available in the online journal.)

also note here that not only do the mean light curves of some Blazhko variables look similar to the light curve of V123, but the same is true for the light curves of some Blazhko stars in a given phase of their modulation.

We have checked which parameters are responsible for the different shapes of the light curves. It is found that the rise time and the higher-order amplitude ratios $\left(R_{k 1}=A_{k} / A_{1}, k>=3\right)$ and phase differences $\left(\Phi_{k 1}=\Phi_{k}-k \Phi_{1}, k>=6\right)$ show the most significant discrepancies for V123 and for the mean light curves of the selected Blazhko stars (see top and middle panels in Figure 2). Based on these parameters, V123 clearly stands out from the very homogeneous population of normal RRab stars; instead, it fits the group of the Blazhko stars. Meanwhile, the total amplitudes of V123 and the Blazhko stars are slightly larger and smaller than the total amplitude of normal RRab stars, respectively.

Another question naturally arises, whether V123 is a bona fide cluster member of M3. The star lies 17" apart from the cluster's center; it is the largest-radial-distance RR Lyrae star of M3. Its membership is, however, $98 \%$ probable, based on the proper motion study by Tucholke et al. (1994). The mean magnitudes and colors, and also the radial-velocity variation of V123, verify this conclusion as documented in the bottom panels of Figure 2 and in Figure 3. The positions of V123 agree within the limits

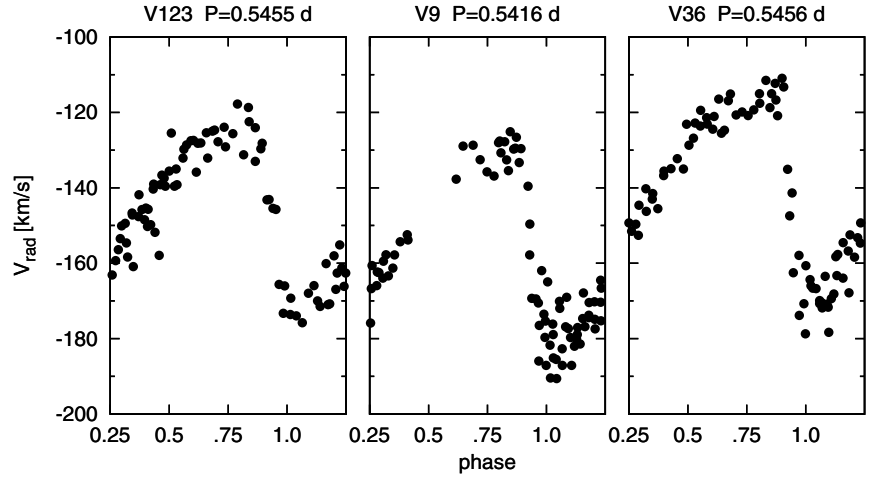

Figure 3. Radial-velocity curves of V123, V9, and V36.
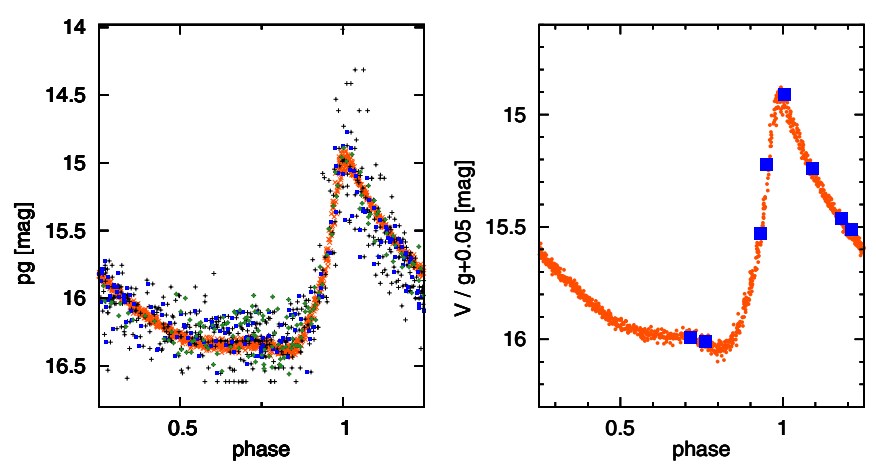

Figure 4. Magnitude zero-point and time-transformed (Jurcsik et al. 2012) archive photographic (pg) light curves of V123 in comparison with the CCD $B$ data (left panel). The pg and CCD data are shown by the "+" and "x" symbols, respectively. Two homogeneous samples of the archive data, the Konkoly observations obtained between 2428900-2437800 and 2438500-2439900 are set out by filled squares and circles. The phase homogenization of the observations takes into account only long-term period changes during the $100 \mathrm{yr}$ of the observations, and does not correct for any possible short-term phase modulation. The comparison of the $2013 \mathrm{~V}$ and the 1999 Thuan-Gunn $g$ (Jurcsik et al. 2012) light curves of V123 is shown in the right panel.

(A color version of this figure is available in the online journal.)

of the uncertainties of the photometry with the positions of not highly evolved, similar-period normal RRab stars in both of the shown color-magnitude diagrams. The mean radial-velocity values of V123, V9, and V36 are also similar, $-146 \mathrm{~km} \mathrm{~s}^{-1}$, $-154 \mathrm{~km} \mathrm{~s}^{-1}$, and $-138 \mathrm{~km} \mathrm{~s}^{-1}$, respectively.

The radial-velocity curve of V123 reflects similar anomalies as its light curve, with longer and less steep variation between the maximum and minimum as other RRab stars display. The full amplitudes of the radial-velocity variations are 50, 55, and $56 \mathrm{~km} \mathrm{~s}^{-1}$ for V123, V9, and V36.

Though V123 was not included in the chemical composition analysis of M3 variables (Sandstrom et al. 2001) because of uncertain $T_{\text {eff }}$ and $\log g$ information due to the sparseness of the photometric data, a rough analysis of the WIYN spectra revealed that its $\mathrm{Fe}, \mathrm{Mg}$, and $\mathrm{Ca}$ abundances are the same within the errors as the abundances of the other variables.

Despite the 200 day coverage of the CCD observations, these data do not exclude large-amplitude modulation on an even longer time scale. The archive photographic data, however, contradict this possibility. They do not indicate any lightcurve variability within the limits of the uncertainty of the data (see Figure 4). The light curve of V123 seemed to be unique with a peculiar-shape rising branch according to the archive photographic data collected in Jurcsik et al. (2012) without any 

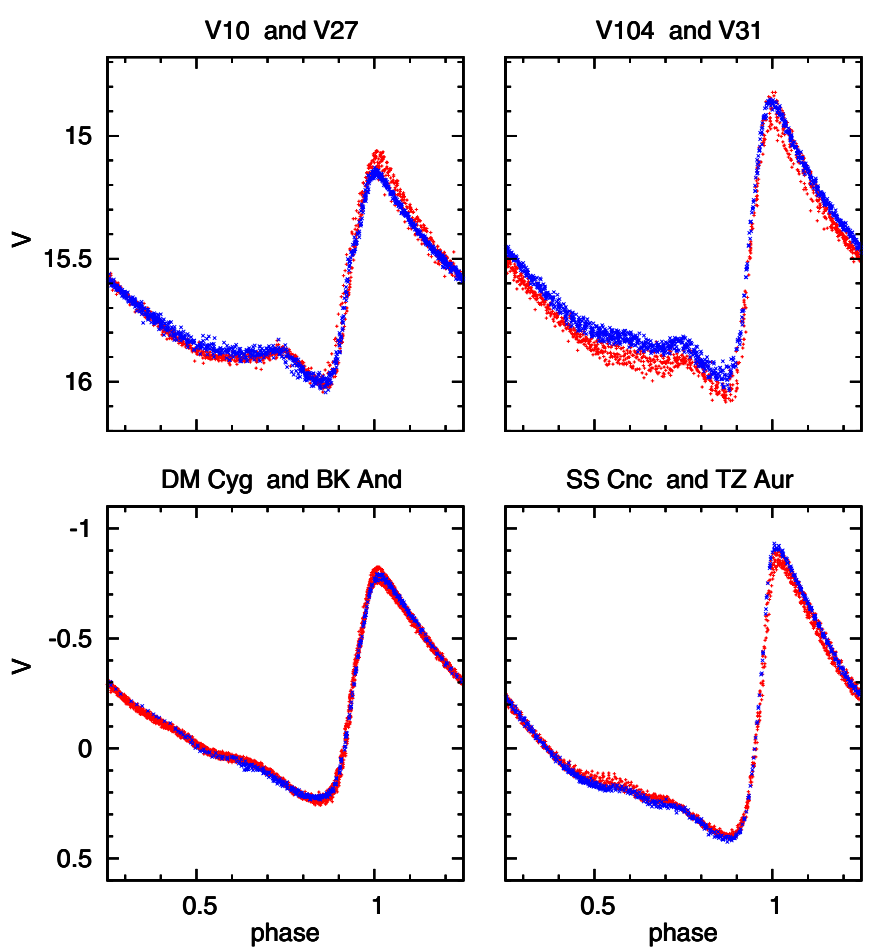

Figure 5. Comparison of four pairs of small-modulation-amplitude Blazhko and regular RRab stars with similar periods in M3 (top-left panel: V10, $P=0.5696$ days and V27, $P=0.5791$ days; top-right panel: V104, $P=0.5699$ days and V31, $P=0.5807$ days) and in the field (bottom-left: DM Cyg, $P=0.4216$ days and BK And, $P=0.4177$ days; bottom-right: SS Cnc, $P=0.3673$ days and TZ Aur, $P=0.3917$ days. Data of field RRab stars are taken from Jurcsik et al. 2009). The mean light curves of small-modulation-amplitude Blazhko stars (red/gray symbols) seem to match fairly well the light curves of regular RRab stars (blue/black symbols). The light curves of field stars are set on an arbitrary magnitude scale.

(A color version of this figure is available in the online journal.)

sign of a strong light-curve modulation (Blazhko effect). The zero-point and phase homogenized light curves of the different archive observations spanning about $100 \mathrm{yr}$ are shown in the left panel of Figure 4. No significant variation in the shape of the light curve is evident, the amplitude differences of the data sets arise mostly from the different magnitude scales of the plate materials utilized. Archive CCD observations of V123 are very sparse, only some Thuan-Gunn uvgri data were published in Jurcsik et al. (2012). The $g$ filter data of V123 match the recent $V$ light curve within the uncertainty limit (right panel in Figure 4), with a 0.05 mag zero-point difference.

We thus conclude that, based on the archive photographic and CCD observations, large-amplitude Blazhko modulation of V123 with a substantially longer modulation cycle than the 200 day interval covered by the recent CCD observations can certainly be ruled out.

V123 is thus either a Blazhko star that exhibits only a smallamplitude, irregular modulation of its anomalous light curve, or it is an anomalous light-curve-shape RRab star showing slight, irregular light-curve fluctuations.

Both the mean and the particular light curves in different phases of the modulation of Blazhko stars are often anomalous. However, the tendency is that the larger the amplitude of the modulation is the more peculiar the light curve can be. Blazhko stars showing small-amplitude modulations look like similarperiod RRab stars of regular type as documented in Figure 5. Consequently, it is unlikely that any small-amplitude, irregular modulation accounts for the anomalous shape of the light curve of V123.

Summarizing:

1. V123 is a cluster member RRab variable of M3 at large radial distance, displaying anomalous-shape light and radialvelocity curves, which resemble the mean variations of Blazhko stars, rather than the variations of similar-period normal RRab stars;

2. its mean magnitudes and colors agree well with the magnitudes and colors of RRab stars with similar pulsation periods;

3. its light curve shows some irregular variations, but no periodic, strong Blazhko modulation is evident.

\section{DISCUSSION}

What kind of object is V123/M3, this anomalous RRab star?

A possible explanation for the similarity of the light curve of V123 and the mean light curves of Blazhko stars might be the aspect-angle dependence of the shapes of the light curves of Blazhko stars during the modulation cycle. In this case, V123 is, in fact, a Blazhko star seen "pole on," with a deformed light-curve shape similar to the mean light curve of a Blazhko star, exhibiting only slight irregularities. In Jurcsik et al. (2005), we have already shown that the Blazhko periods, if converted to rotational velocities, do not contradict the observed $v \sin i$ distribution of HB stars. The upper limit for the $v \sin i$ rotational velocities of Blazhko stars defined by spectroscopic studies ( $\sim 10 \mathrm{~km} \mathrm{~s}^{-1}$; Peterson et al. 1996; Chadid \& Preston 2013) is expected to be exceeded only in the case of extremely short modulation period Blazhko stars $(P<5$ days $)$ with inclination close to $90^{\circ}$. These stars are located close to the fundamental blue edge, as short modulation periods are detected only among short pulsation-period variables (Jurcsik et al. 2005).

Such a scenario would mean that the spherical symmetry and/or the homogeneity of Blazhko stars have to be broken. This can be caused by, e.g., magnetic activity, chemical inhomogeneity, or nonradial pulsation modes. Although no surface magnetic field of Blazhko stars are detected by recent observations, the deep magnetic field of RR Lyrae stars may remain unobserved according to Stothers (2006). Observations of the prototype RR Lyr by Chadid et al. (2004) showed no evidence for a strong magnetic field in the star's photosphere, and dismissed the hypothesis by Stothers (1980) that RR Lyr undergoes a magnetic cycle. However, more complex morphologies of a surface magnetic field may remain undetectable with current instrumentation (see Kolenberg \& Bagnulo 2009). We may also speculate that engulfing small companions (cool dwarf stars or planets) on the tip of the giant branch may result in surface chemical inhomogeneities in the early stages of the HB evolution. It is important to note that a detailed high-dispersion spectroscopic and photometric study revealed that TY Gru, a large-modulation-amplitude Blazhko RRab star, shows large over-abundances of carbon and neutron-capture elements, most probably due to mass transfer from an asymptotic giant branch companion (Preston et al. 2006). However, no evidence for either a systematic chemical composition difference between Blazhko and non-Blazhko stars or between the observed chemical compositions of Blazhko stars in different phases of the modulation were detected in a detailed spectroscopic study of Blazhko and non-Blazhko stars (For et al. 2011). Finally, a strong deformation of the main radial pulsation mode by nonradial pulsation components, such as that described in the magnetic model by 
Shibahashi (2000), can also contribute to breaking the spherical symmetry of an RR Lyrae star. In a "pole-on" configuration, this model results in light variation of a somewhat anomalous shape without any sign of multiplet components in its Fourier spectrum.

As the light curves of some Blazhko stars during their modulations are temporarily also similar to the light curve of V123, its anomalous behavior might also be interpreted as being a Blazhko star, with its pulsation locked in one special Blazhko phase. The explanation of the distorted pulsation curves of Blazhko stars during their modulation cycle might be thus the same as for the anomalous light-curve shape of V123.

If V123 is neither a "pole-on" nor a "phase-locked" Blazhko star, we have to find the reason why the shape of its light curve is anomalous. As its mean brightness, colors and Fe, $\mathrm{Mg}$, and $\mathrm{Ca}$ abundances are equal within the error margin with the brightness, colors, and the abundances of non-evolved regular RRab stars with similar periods in M3, its luminosity and temperature, and as a consequence its mass should not differ significantly from those of other RRab stars. To change the hydrodynamics while maintaining the same main physical properties, we are left again with the fact that the abundances of other chemical compositions of the star have to be changed significantly to reach any detectable light-curve anomaly. This can happen if the evolutionary history of V123 differs from that of other RRab stars. Based on the observed properties, we can exclude, however, the possibility that an evolutionary scenario involving significant mass exchange of a binary system (e.g., in Pietrzynski et al. 2012) is behind the phenomenon. If V123 were still a member of a binary system, the secondary would have to be a very low-luminosity object, with no measurable influence on the total luminosity of the system. However, such a companion cannot affect the light-curve shape significantly. If an anomalous chemical composition is behind the aberrant-lightcurve shape of V123, the only plausible explanation remains the contamination of the atmosphere by the capture of a very small mass object during the evolution on the giant branch. The result of this scenario is, however, most probably an extreme-HB star instead of an RR Lyr (Bear \& Soker 2011).

Whatever the solution for V123, its similarity to Blazhko stars suggests that maybe the same mechanism influences the hydrodynamics and the triggering/damping mechanism of their pulsations, consequently it can be a key object in resolving the Blazhko phenomenon. High-dispersion spectroscopic observations, and hydrodynamical modeling of the anomalous light curve of V123 are needed to reveal the true nature of this peculiar object.

This Letter is dedicated to the memory of Professor Béla Szeidl, former director of Konkoly Observatory, whose life-long interest on the Blazhko modulation motivated many successful studies.

G.H. gratefully acknowledges support from the Chilean Ministry for the Economy, Development, and Tourisms Programa Iniciativa Cientfica Milenio through grant P07-021-F, awarded to the Milky Way Millennium Nucleus. WIYN observations were obtained through the WIYN Queue Program by Paul Smith and Daryl Willmarth, with assistance from G. Rosenstein and W. Hughes; their contributions are gratefully acknowledged. C.A.P. acknowledges the generosity of the Kirkwood Research Fund at Indiana University. K.K. is grateful for the support from a Marie Curie Fellowship (255267 SAS-RRL) within the 7th European Community Framework Programme (FP7). Á.S. acknowledges support from the Belgian Federal Science Policy (project M0/33/029).

\section{REFERENCES}

Bear, E., \& Soker, N. 2011, MNRAS, 411, 1792

Blazhko, S. 1907, AN, 175, 327

Chadid, M., \& Preston, G. W. 2013, MNRAS, 434, 552

Chadid, M., Wade, G. A., Shorlin, S. L. S., \& Landstreet, J. D. 2004, A\&A, 413, 1087

Fitch, W. S., \& Szeidl, B. 1976, ApJ, 203, 616

For, B.-Q., Sneden, C., \& Preston, G. W. 2011, ApJS, 197, 1

Jurcsik, J. 1998, A\&A, 333, 571

Jurcsik, J., Hajdu, G., Szeidl, B., et al. 2012, MNRAS, 419, 2173

Jurcsik, J., \& Kovács, G. 1996, A\&A, 312, 111

Jurcsik, J., Sódor, Á., Szeidl, B., et al. 2009, MNRAS, 400, 1006

Jurcsik, J., Szeidl, B., Nagy, A., \& Sódor, Á. 2005, AcA, 55, 303

Kolenberg, K., \& Bagnulo, S. 2009, A\&A, 498, 543

Kovács, G., \& Buchler, R. 1994, A\&A, 281, 749

Kovács, G., \& Walker, A. 2001, A\&A, 371, 579

Marconi, M., \& Degl'Innocenti, S. 2007, A\&A, 474, 557

Peterson, R. C., Carney, B. W., \& Latham, D. W. 1996, ApJL, 465, L47

Pietrzynski, G., Thompson, I. B., Gieren, W., et al. 2012, Natur, 484, 75

Preston, G. W., Thompson, I. B., Sneden, C., Stachowski, G., \& Shectman, S. A. 2006, AJ, 132, 1714

Sandstrom, K., Pilachowski, C. A., \& Saha, A. 2001, AJ, 122, 3212

Shibahashi, H. 2000, in ASP Conf. Ser. 203, The Impact of Large-Scale Surveys on Pulsating Star Research, ed. L. Szabados \& D. Kurtz. (San Francisco, CA: ASP), 299

Stothers, R. 1980, ApJ, 242, 756

Stothers, R. 2006, ApJL, 655, L63

Tucholke, H.-J., Scholz, R.-D., \& Brosche, P. 1994, A\&AS, 104, 161 